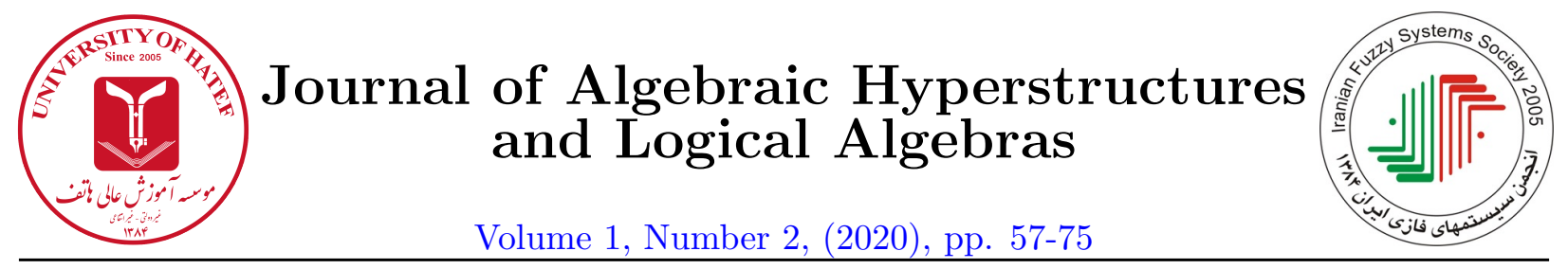

\title{
MBJ-neutrosophic filters of equality algebras
}

\author{
M. Mohseni Takallo ${ }^{1}$ and M. Aaly Kologani ${ }^{2}$ \\ ${ }^{1}$ Department of Mathematics, Shahid Beheshti University, Tehran, Iran \\ ${ }^{2}$ Hatef Higher Education Institute, Zahedan, Iran
}

mohammad.mohseni1122@gmail.com, mona4011@gmail.com

\begin{abstract}
In this paper, we introduce the notion of MBJneutrosophic sub-algebra and MBJ-neutrosophic filter on equality algebras and investigate some equivalence definitions, properties and the relation between them. Also, by using the notion of MBJ-neutrosophic filter, we introduce a congruence relation on equality algebra and show that the quotient is an equality algebra.
\end{abstract}

\section{Article Information}

Corresponding Author:

M. Aaly Kologani;

Received: May 2020;

Accepted: May 2020.

Paper type: Original.

\section{Keywords:}

MBJ-neutrosophic set, MBJneutrosophic filter, equality algebra, filter.

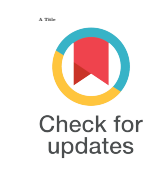

\section{Introduction}

Non-classical logic has become a considerable formal tool for computer science and artificial intelligence to deal with fuzzy information and uncertainty information. Many-valued logic, a great extension and development of classical logic, has always been a crucial direction in non-classical logic. A crucial question for every many-valued logic is, what should be structure of its truth values. It is generally accepted that in fuzzy logic, it should be a residuated lattice, possibly fulfilling some additional properties. On the basis of that, we may now distinguish various kinds of formal fuzzy logics. Most important among them seem to be BL-, MTL-, IMTL- logics. The answer to the above question is positive and the fuzzy type theory (FTT) has indeed been introduced in [20]. However, the basic connective in FTT is a fuzzy equality since it is developed as a generalization of the elegant classical formal system originated by Henkin (see [7]). So Novák in [21] introduced a special algebra that is called EQ-algebra and that reflects directly the syntax of FTT. Viewing the axioms of EQ-algebras with a purely algebraic eye it appears that unlike in the case of residuated

https://doi.org/10.29252/HATEF.JAHLA.1.2.6 
lattices where the adjointness condition ties product with implication, the product in EQ-algebras is quite loosely related to the other connectives. For instance, a moments reflection shows that one can replace the product of an EQ-algebra by any other binary operation which is smaller or equal than the original product (viewed as a two-place function) and still obtains an EQ-algebra. However, the huge freedom in choosing the product might prohibit to find deep related algebraic results, hence our aim was to find something similar to EQ-algebras but without a product: an axiomatic treatment of equality/equivalence. Becuase of that Jeni in [10] introduced a new structure, called equality algebras. It has two connectives, a meet operation and an equivalence, and a constant 1 .

Neutrosophic set theory was introduced by Smarandache in 1998 ([23]). Neutrosophic sets are a new mathematical tool for dealing with uncertainties which are free from many difficulties that have troubled the usual theoretical approaches. Research works on neutrosophic set theory for many applications such as information fussion, probability theory, control theory, decision making, measurement theory, etc. Kandasamy and Smarandache introduced the concept of neutrosophic algebraic structures $([15,16])$. Since then many researchers worked in this area and lots of literatures had been produced about the theory of neutrosophic set. The notion of MBJ-neutrosophic sets is introduced as another generalization of neutrosophic set, it is applied to BCK/BCI-algebras. Mohseni et al.[19] introduced the concept of MBJ-neutrosophic subalgebras in BCK/BCI-algebras, and investigated related properties. They gave a characterization of MBJ-neutrosophic subalgebra, and established a new MBJ-neutrosophic subalgebra by using an MBJ-neutrosophic subalgebra of a BCI-algebra. They considered the homomorphic inverse image of MBJ-neutrosophic subalgebra, and discussed translation of MBJ-neutrosophic subalgebra.

In this paper, the notion of MBJ-neutrosophic filter on equality algebras is introduced and investigated some equivalence definitions and some properties of it. Also, by using the notion of MBJ-neutrosophic filter, a congruence relation on equality algebra is introduced.

\section{Preliminaries}

In this section, we give some basic definitions and results of equality algebras which will be used in this paper.

Definition 2.1. 9] An algebraic structure $(X, \wedge, \sim, 1)$ is called equality algebra, if for any $a, b, c \in$ $X$, it satisfies the following conditions.

(E1) $(X, \wedge, 1)$ is a commutative idempotent integral monoid,

(E2) the operation " $\sim$ " is commutative,

(E3) $a \sim a=1$,

(E4) $a \sim 1=a$,

(E5) if $a \leq b \leq c$, then $a \sim c \leq b \sim c$ and $a \sim c \leq a \sim b$,

(E6) $a \sim b \leq(a \wedge c) \sim(b \wedge c)$,

(E7) $a \sim b \leq(a \sim c) \sim(b \sim c)$,

where $a \leq b$ if and only if $a \wedge b=a$. 
In an equality algebra $X=(X, \wedge, \sim, 1)$, we define two operations " $\rightarrow$ " and " $\leftrightarrow$ " on $X$ as follows:

$$
a \rightarrow b:=a \sim(a \wedge b) \text { and } a \leftrightarrow b:=(a \rightarrow b) \wedge(b \rightarrow a) .
$$

Proposition 2.2. 9] Let $X:=(X, \wedge, \sim, 1)$ be an equality algebra. Then for any $a, b, c \in X$, the following assertions are valid:

(i) $a \rightarrow b=1$ if and only if $a \leq b$,

(ii) $a \rightarrow(b \rightarrow c)=b \rightarrow(a \rightarrow c)$,

(iii) $1 \rightarrow a=a, a \rightarrow 1=1$ and $a \rightarrow a=1$,

(iv) $a \leq b \rightarrow c$ if and only if $b \leq a \rightarrow c$,

(v) $a \leq b \rightarrow a$,

(vi) $a \leq(a \rightarrow b) \rightarrow b$,

(vii) $a \rightarrow b \leq(b \rightarrow c) \rightarrow(a \rightarrow c)$,

(viii) If $b \leq a$, then $a \leftrightarrow b=a \rightarrow b=a \sim b$,

(ix) $a \sim b \leq a$ if and only if $b \leq a \rightarrow b$,

(x) If $a \leq b$, then $b \rightarrow c \leq a \rightarrow c$ and $c \rightarrow a \leq c \rightarrow b$.

An equality algebra $X:=(X, \wedge, \sim, 1)$ is said to be bounded if there exists an element $0 \in X$ such that $0 \leq a$, for all $a \in X$. In a bounded equality algebra $X:=(X, \wedge, \sim, 1)$, we define the negation " $\neg$ " on $X$ by $\neg a=a \rightarrow 0=a \sim 0$ for all $a \in X$.

A subset $F$ of $X$ is called a deductive system (or filter) of $X$ (see [10]) if for any $a, b \in X$, it satisfies:

$\left(F_{1}\right) \quad 1 \in F$,

$\left(F_{2}\right)$ if $a \in F$ and $a \leq b$, then $b \in F$,

$\left(F_{3}\right)$ if $a \in F$ and $a \sim b \in F$, then $b \in F$.

The set of all deductive systems of $X$ is denoted by $\mathcal{D S}(X)$.

Lemma 2.3. [8] Let $X$ be an equality algebra. A subset $F$ of $X$ is called a deductive system of $X$ if and only if it satisfies $\left(F_{1}\right)$ and for any $a, b \in X$,

$(F) \quad$ if $a \in F$ and $a \rightarrow b \in F$, then $b \in F$.

Definition 2.4. [27] An equality algebra $X=(X, \wedge, \sim, 1)$ is called a commutative if for any $a, b \in X,(a \rightarrow b) \rightarrow b=(b \rightarrow a) \rightarrow a$.

Definition 2.5. [1] An equality algebra $X=(X, \wedge, \sim, 1)$ is called a positive implicative if for any $a, b, c \in X, a \rightarrow(b \rightarrow c)=(a \rightarrow b) \rightarrow(a \rightarrow c)$.

By an interval number we mean a closed subinterval $\tilde{a}=\left[a^{-}, a^{+}\right]$of $I=[0,1]$, where $0 \leq a^{-} \leq$ $a^{+} \leq 1$. Denote by $[I]$ the set of all interval numbers. Let us define what is known as refined minimum (briefly, rmin) and refined maximum (briefly, rmax) of two elements in $[I]$. We also define the symbols " $\succeq$ ", "£", "=" in case of two elements in $[I]$. Consider two interval numbers $\tilde{a}_{1}:=\left[a_{1}^{-}, a_{1}^{+}\right]$and $\tilde{a}_{2}:=\left[a_{2}^{-}, a_{2}^{+}\right]$. Then

$$
\begin{aligned}
& \operatorname{rmin}\left\{\tilde{a}_{1}, \tilde{a}_{2}\right\}=\left[\min \left\{a_{1}^{-}, a_{2}^{-}\right\}, \min \left\{a_{1}^{+}, a_{2}^{+}\right\}\right], \\
& \operatorname{rmax}\left\{\tilde{a}_{1}, \tilde{a}_{2}\right\}=\left[\max \left\{a_{1}^{-}, a_{2}^{-}\right\}, \max \left\{a_{1}^{+}, a_{2}^{+}\right\}\right], \\
& \tilde{a}_{1} \succeq \tilde{a}_{2} \Leftrightarrow a_{1}^{-} \geq a_{2}^{-}, a_{1}^{+} \geq a_{2}^{+},
\end{aligned}
$$

and similarly we may have $\tilde{a}_{1} \preceq \tilde{a}_{2}$ and $\tilde{a}_{1}=\tilde{a}_{2}$. To say $\tilde{a}_{1} \succ \tilde{a}_{2}$ (resp. $\left.\tilde{a}_{1} \prec \tilde{a}_{2}\right)$ we mean $\tilde{a}_{1} \succeq \tilde{a}_{2}$ and $\tilde{a}_{1} \neq \tilde{a}_{2}$ (resp. $\tilde{a}_{1} \preceq \tilde{a}_{2}$ and $\tilde{a}_{1} \neq \tilde{a}_{2}$ ). Let $\tilde{a}_{i} \in[I]$ where $i \in \Lambda$. We define

$$
\operatorname{rinf}_{i \in \Lambda} \tilde{a}_{i}=\left[\inf _{i \in \Lambda} a_{i}^{-}, \inf _{i \in \Lambda} a_{i}^{+}\right] \text {and } \operatorname{rsup}_{i \in \Lambda} \tilde{a}_{i}=\left[\sup _{i \in \Lambda} a_{i}^{-}, \sup _{i \in \Lambda} a_{i}^{+}\right] .
$$


Let $X$ be a nonempty set. A function $A: X \rightarrow[I]$ is called an interval-valued fuzzy set (briefly, an $I V F$ set) in $X$. Let $[I]^{X}$ stand for the set of all IVF sets in $X$. For every $A \in[I]^{X}$ and $x \in X$, $A(x)=\left[A^{-}(x), A^{+}(x)\right]$ is called the degree of membership of an element $x$ to $A$, where $A^{-}: X \rightarrow I$ and $A^{+}: X \rightarrow I$ are fuzzy sets in $X$ which are called a lower fuzzy set and an upper fuzzy set in $X$, respectively. For simplicity, we denote $A=\left[A^{-}, A^{+}\right]$.

Let $X$ be a non-empty set. A neutrosophic set (NS) in $X$ (see [24]) is a structure of the form:

$$
A:=\left\{\left\langle x ; A_{T}(x), A_{I}(x), A_{F}(x)\right\rangle \mid x \in X\right\}
$$

where $A_{T}: X \rightarrow[0,1]$ is a truth membership function, $A_{I}: X \rightarrow[0,1]$ is an indeterminate membership function, and $A_{F}: X \rightarrow[0,1]$ is a false membership function.

Let $X$ be a non-empty set. By an MBJ-neutrosophic set in $X$ (see [19]), we mean a structure of the form:

$$
\mathcal{A}:=\left\{\left\langle x ; M_{A}(x), \tilde{B}_{A}(x), J_{A}(x)\right\rangle \mid x \in X\right\},
$$

where $M_{A}$ and $J_{A}$ are fuzzy sets in $X$, which are called a truth membership function and a false membership function, respectively, and $\tilde{B}_{A}$ is an IVF set in $X$ which is called an indeterminate interval-valued membership function.

We use the symbol $\mathcal{A}=\left(M_{A}, \tilde{B}_{A}, J_{A}\right)$ for the MBJ-neutrosophic set

$$
\mathcal{A}:=\left\{\left\langle x ; M_{A}(x), \tilde{B}_{A}(x), J_{A}(x)\right\rangle \mid x \in X\right\} .
$$

Note. From now on, we let $X=(X, \wedge, \sim, 1)$ or $X$ as an equality algebra unless otherwise state.

\section{MBJ-neutrosophic sub-algberas and filters}

In this section we define the notion of MBJ-neutrosophic sub-algebra and MBJ-neutrosophic filter of $X$ and investigate some equivalence definitions and some propoerties of them.

Definition 3.1. An $M B J$-neutrosophic set $\mathcal{A}=\left(M_{A}, \tilde{B}_{A}, J_{A}\right)$ in $X$ is called an MBJ-neutrosophic sub-algebra of $X$ if it satisfies

$$
(\forall x, y, z \in X)\left(\begin{array}{l}
M_{A}(x \sim y) \geq \min \left\{M_{A}(x), M_{A}(y)\right\} \\
\tilde{B}_{A}(x \sim y) \succeq \operatorname{rmin}\left\{\tilde{B}_{A}(x), \tilde{B}_{A}(y)\right\} \\
J_{A}(x \sim y) \leq \max \left\{J_{A}(x), J_{A}(y)\right\}
\end{array}\right),
$$

and

$$
(\forall x, y, z \in X)\left(\begin{array}{l}
M_{A}(x \wedge y) \geq \min \left\{M_{A}(x), M_{A}(y)\right\} \\
\tilde{B}_{A}(x \wedge y) \succeq \operatorname{rmin}\left\{\tilde{B}_{A}(x), \tilde{B}_{A}(y)\right\} \\
J_{A}(x \wedge y) \leq \max \left\{J_{A}(x), J_{A}(y)\right\}
\end{array}\right)
$$

Example 3.2. Let $X=\{0, a, b, 1\}$ be a set with the following Hasse diagram.

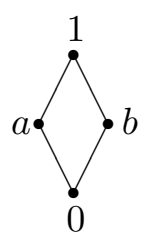


Then $(X, \wedge, 1)$ is a commutative idempotent integral monoid. We define a binary operation $\sim$ on $X$ by Table 1 .

\begin{tabular}{lllll}
\hline$\sim$ & 0 & $a$ & $b$ & 1 \\
\hline 0 & 1 & $b$ & $a$ & 0 \\
$a$ & $b$ & 1 & 0 & $a$ \\
$b$ & $a$ & 0 & 1 & $b$ \\
1 & 0 & $a$ & $b$ & 1 \\
\hline
\end{tabular}

Table 1: Cayley table for the implication " $"$

Then $\mathcal{E}=(X, \wedge, \sim, 1)$ is an equality algebra. Let define $\mathcal{A}=\left(M_{A}, \tilde{B}_{A}, J_{A}\right)$ in $X$ as Table 2.

\begin{tabular}{cccc}
\hline$X$ & $M_{A}$ & $\tilde{B}_{A}$ & $J_{A}$ \\
\hline 0 & 0.4 & {$[0.4,0.5]$} & 0.5 \\
$a$ & 0.5 & {$[0.3,0.45]$} & 0.5 \\
$b$ & 0.4 & {$[0.3,0.45]$} & 0.5 \\
1 & 0.7 & {$[0.7,0.8]$} & 0.2 \\
\hline
\end{tabular}

Table 2: Cayley table for the implication " $\mathcal{A}$ "

By routine calculation, we can see that $\mathcal{A}=\left(M_{A}, \tilde{B}_{A}, J_{A}\right)$ is an MBJ-neutrosophic sub-algebra of $X$.

Proposition 3.3. If $\mathcal{A}=\left(M_{A}, \tilde{B}_{A}, J_{A}\right)$ is an $M B J$-neutrosophic sub-algebra of $X$, then $M_{A}(1) \geq$ $M_{A}(x), \tilde{B}_{A}(1) \succeq \tilde{B}_{A}(x)$ and $J_{A}(1) \leq J_{A}(x)$, for all $x \in X$.

Proof. For any $x \in X$, we get

$$
\begin{gathered}
M_{A}(1)=M_{A}(x \sim x) \geq \min \left\{M_{A}(x), M_{A}(x)\right\}=M_{A}(x), \\
\tilde{B}_{A}(1)=\tilde{B}_{A}(x \sim x) \succeq \operatorname{rmin}\left\{\tilde{B}_{A}(x), \tilde{B}_{A}(x)\right\}=\tilde{B}_{A}(x),
\end{gathered}
$$

and

$$
J_{A}(1)=J_{A}(x \sim x) \leq \max \left\{J_{A}(x), J_{A}(x)\right\}=J_{A}(x)
$$

In the following example we show that the converse of above proposition may not be true, in general.

Example 3.4. Let $X$ be the equality algebra as Example 3.2. Define $\mathcal{A}=\left(M_{A}, \tilde{B}_{A}, J_{A}\right)$ in $X$ as Table 3.

\begin{tabular}{cccc}
\hline$X$ & $M_{A}$ & $\tilde{B}_{A}$ & $J_{A}$ \\
\hline 0 & 0.4 & {$[0.2,0.3]$} & 0.5 \\
$a$ & 0.5 & {$[0.4,0.5]$} & 0.5 \\
$b$ & 0.4 & {$[0.5,0.6]$} & 0.5 \\
1 & 0.7 & {$[0.7,0.8]$} & 0.2 \\
\hline
\end{tabular}

Table 3: Cayley table for the implication " $\mathcal{A}$ " 
It is clear that for all $x \in X, M_{A}(1) \geq M_{A}(x), \tilde{B}_{A}(1) \succeq \tilde{B}_{A}(x)$ and $J_{A}(1) \leq J_{A}(x)$. But $\mathcal{A}=\left(M_{A}, \tilde{B}_{A}, J_{A}\right)$ is not an MBJ-neutrosophic sub-algebra of $X$, because

$$
[0.2,0.3]=\tilde{B}_{A}(0)=\tilde{B}_{A}(a \wedge b) \nsucceq \operatorname{rmin}\left\{\tilde{B}_{A}(a), \tilde{B}_{A}(b)\right\}=\tilde{B}_{A}(a)=[0.4,0.5] .
$$

Theorem 3.5. Let $\mathcal{A}=\left(M_{A}, \tilde{B}_{A}, J_{A}\right)$ be an MBJ-neutrosophic sub-algebra of $X$. Then the following staements hold:

$$
(\forall x, y \in X)\left(\begin{array}{l}
M_{A}(x \rightarrow y) \geq \min \left\{M_{A}(x), M_{A}(y)\right\} \\
\tilde{B}_{A}(x \rightarrow y) \succeq \operatorname{rmin}\left\{\tilde{B}_{A}(x), \tilde{B}_{A}(y)\right\} \\
J_{A}(x \rightarrow y) \leq \max \left\{J_{A}(x), J_{A}(y)\right\}
\end{array}\right)
$$

Proof. Let $\mathcal{A}=\left(M_{A}, \tilde{B}_{A}, J_{A}\right)$ be an MBJ-neutrosophic sub-algebra of $X$ and $x, y \in X$. Then by Definition 3.1. we have

$$
\begin{aligned}
& M_{A}(x \rightarrow y)=M_{A}(x \sim(x \wedge y)) \geq \min \left\{M_{A}(x), M_{A}(x \wedge y)\right\} \geq \min \left\{M_{A}(x), M_{A}(y)\right\} \\
& \tilde{B}_{A}(x \rightarrow y)=\tilde{B}_{A}(x \sim(x \wedge y)) \succeq \operatorname{rmin}\left\{\tilde{B}_{A}(x), \tilde{B}_{A}(x \wedge y)\right\} \succeq \operatorname{rmin}\left\{\tilde{B}_{A}(x), \tilde{B}_{A}(y)\right\} \\
& J_{A}(x \rightarrow y)=J_{A}(x \sim(x \wedge y)) \leq \max \left\{J_{A}(x), J_{A}(x \wedge y)\right\} \leq \max \left\{J_{A}(x), J_{A}(y)\right\}
\end{aligned}
$$

Hence (3.3) holds.

In the following example we show that the converse of Theorem 3.5 may not be true, in general.

Example 3.6. Let $X$ be the equality algebra as Example 3.2. It is clear that the table of implication is as Table 4:

\begin{tabular}{ccccc}
\hline$\rightarrow$ & 0 & $a$ & $b$ & 1 \\
\hline 0 & 1 & 1 & 1 & 1 \\
$a$ & $b$ & 1 & $b$ & 1 \\
$b$ & $a$ & $a$ & 1 & 1 \\
1 & 0 & $a$ & $b$ & 1 \\
\hline
\end{tabular}

Table 4: Cayley table for the implication " $\rightarrow$ "

Define $\mathcal{A}=\left(M_{A}, \tilde{B}_{A}, J_{A}\right)$ in $X$ as Table 5 .

\begin{tabular}{cccc}
\hline$X$ & $M_{A}$ & $\tilde{B}_{A}$ & $J_{A}$ \\
\hline 0 & 0.3 & {$[0.4,0.5]$} & 0.5 \\
$a$ & 0.5 & {$[0.3,0.45]$} & 0.5 \\
$b$ & 0.4 & {$[0.3,0.45]$} & 0.5 \\
1 & 0.7 & {$[0.7,0.8]$} & 0.2 \\
\hline
\end{tabular}

Table 5: Cayley table for the implication " $\mathcal{A}$ "

Routine calculation show that the condition (3.3) holds but $\mathcal{A}=\left(M_{A}, \tilde{B}_{A}, J_{A}\right)$ is not an $M B J$-neutrosophic sub-algebra of $X$, because

$$
0.3=M_{A}(0)=M_{A}(a \wedge b) \ngtr \min \left\{M_{A}(a), M_{A}(b)\right\}=M_{A}(b)=0.4 .
$$


Theorem 3.7. Let $X$ be a linearly order equality algebra and $\mathcal{A}=\left(M_{A}, \tilde{B}_{A}, J_{A}\right)$ be an $M B J$ neutrosophic set in $X$ which satisfies in condition (3.3). Then $\mathcal{A}=\left(M_{A}, \tilde{B}_{A}, J_{A}\right)$ is an $M B J$ neutrosophic sub-algebra in $X$.

Proof. Let $x, y \in X$ and $\mathcal{A}=\left(M_{A}, \tilde{B}_{A}, J_{A}\right)$ be an MBJ-neutrosophic set in $X$. Since $X$ is a linearly order equality algebra, we get $x \leq y$ or $y \leq x$. Suppose $x \leq y$. Then by Proposition 2.2 (viii) and (E2), we have $y \rightarrow x=y \sim x=x \sim y$. Thus

$$
\begin{aligned}
& M_{A}(x \sim y)=M_{A}(y \rightarrow x) \geq \min \left\{M_{A}(x), M_{A}(y)\right\}, \\
& \tilde{B}_{A}(x \sim y)=\tilde{B}_{A}(y \rightarrow x) \succeq \operatorname{rmin}\left\{\tilde{B}_{A}(x), \tilde{B}_{A}(y)\right\}, \\
& J_{A}(x \sim y)=J_{A}(y \rightarrow x) \leq \max \left\{J_{A}(x), J_{A}(y)\right\} .
\end{aligned}
$$

Moreover, since $x \leq y$, we have $x \wedge y=x$ and it is clear that

$$
\begin{aligned}
& M_{A}(x \wedge y) \geq \min \left\{M_{A}(x), M_{A}(y)\right\}, \\
& \tilde{B}_{A}(x \wedge y) \succeq \operatorname{rmin}\left\{\tilde{B}_{A}(x), \tilde{B}_{A}(y)\right\}, \\
& J_{A}(x \wedge y) \leq \max \left\{J_{A}(x), J_{A}(y)\right\} .
\end{aligned}
$$

Therefore, $\mathcal{A}=\left(M_{A}, \tilde{B}_{A}, J_{A}\right)$ is an MBJ-neutrosophic sub-algebra in $X$.

In the following we introduce the notion of MBJ-neutrosophic filters of equality algebras.

Definition 3.8. An MBJ-neutrosophic set $\mathcal{A}=\left(M_{A}, \tilde{B}_{A}, J_{A}\right)$ in $X$ is called an MBJ-neutrosophic filter of $X$ if it satisfies

$$
\begin{gathered}
(\forall x \in X)\left(M_{A}(x) \leq M_{A}(1), \tilde{B}_{A}(x) \preceq \tilde{B}_{A}(1), J_{A}(x) \geq J_{A}(1)\right), \\
(\forall x, y, z \in X)\left(\begin{array}{l}
M_{A}(y) \geq \min \left\{M_{A}(x), M_{A}(x \sim y)\right\} \\
\tilde{B}_{A}(y) \succeq \operatorname{rmin}\left\{\tilde{B}_{A}(x), \tilde{B}_{A}(x \sim y)\right\} \\
J_{A}(y) \leq \max \left\{J_{A}(x), J_{A}(x \sim y)\right\}
\end{array}\right),
\end{gathered}
$$

and

$$
(\forall x, y \in X)\left(x \leq y \Rightarrow\left\{\begin{array}{l}
M_{A}(y) \geq M_{A}(x) \\
\tilde{B}_{A}(y) \succeq \tilde{B}_{A}(x) \\
J_{A}(y) \leq J_{A}(x)
\end{array}\right)\right.
$$

Example 3.9. Let $X$ be the equality algebra as Example 3.2. Define $\mathcal{A}=\left(M_{A}, \tilde{B}_{A}, J_{A}\right)$ in $X$ as Table 6 .

\begin{tabular}{cccc}
\hline$X$ & $M_{A}$ & $\tilde{B}_{A}$ & $J_{A}$ \\
\hline 0 & 0.4 & {$[0.3,0.4]$} & 0.6 \\
$a$ & 0.5 & {$[0.4,0.5]$} & 0.4 \\
$b$ & 0.4 & {$[0.4,0.5]$} & 0.5 \\
1 & 0.7 & {$[0.7,0.8]$} & 0.2 \\
\hline
\end{tabular}

Table 6: Cayley table for the implication " $\mathcal{A}$ "

Then routine calculations show that $\mathcal{A}=\left(M_{A}, \tilde{B}_{A}, J_{A}\right)$ is an MBJ-neutrosophic filter in $X$. 
Now, in the follow, we discuss characterizations of an MBJ-neutrosophic filter;

Theorem 3.10. Let $\mathcal{A}=\left(M_{A}, \tilde{B}_{A}, J_{A}\right)$ be an MBJ-neutrosophic set in $X$. Then $\mathcal{A}$ is an $M B J$ neutrosophic filter of $X$ if and only if it satisfies (3.4) and the following condition:

$$
(\forall x, y \in X)\left(\begin{array}{l}
M_{A}(y) \geq \min \left\{M_{A}(x), M_{A}(x \rightarrow y)\right\} \\
\tilde{B}_{A}(y) \succeq \operatorname{rmin}\left\{\tilde{B}_{A}(x), \tilde{B}_{A}(x \rightarrow y)\right\} \\
J_{A}(y) \leq \max \left\{J_{A}(x), J_{A}(x \rightarrow y)\right\}
\end{array}\right) .
$$

Proof. Assume that $\mathcal{A}$ is an MBJ-neutrosophic filter of $X$. It is clear that the condition (3.4) holds. Let $x, y \in X$. By Definition 3.8, for all $x, y \in X$, we get

$$
\begin{aligned}
& \min \left\{M_{A}(x), M_{A}(x \rightarrow y)\right\}=\min \left\{M_{A}(x), M_{A}(x \sim(x \wedge y))\right\} \leq M_{A}(x \wedge y) \leq M_{A}(y), \\
& \operatorname{rmin}\left\{\tilde{B}_{A}(x), \tilde{B}_{A}(x \rightarrow y)\right\}=\operatorname{rmin}\left\{\tilde{B}_{A}(x), \tilde{B}_{A}(x \sim(x \wedge y))\right\} \preceq \tilde{B}_{A}(x \wedge y) \preceq \tilde{B}_{A}(y), \\
& \max \left\{J_{A}(x), J_{A}(x \rightarrow y)\right\}=\max \left\{J_{A}(x), J_{A}(x \sim(x \wedge y))\right\} \geq J_{A}(x \wedge y) \geq J_{A}(y) .
\end{aligned}
$$

Hence, for all $x, y \in X$, we have

$$
\begin{aligned}
& M_{A}(y) \geq \min \left\{M_{A}(x), M_{A}(x \rightarrow y)\right\} \\
& \tilde{B}_{A}(y) \succeq \operatorname{rmin}\left\{\tilde{B}_{A}(x), \tilde{B}_{A}(x \rightarrow y)\right\} \\
& J_{A}(y) \leq \max \left\{J_{A}(x), J_{A}(x \rightarrow y)\right\}
\end{aligned}
$$

Conversly, suppose that $\mathcal{A}$ satisfies in (3.4) and (3.7). Let $x, y \in X$. If $x \leq y$, then $x \rightarrow y=1$ and by (3.4) and (3.7), we get

$$
\begin{aligned}
& M_{A}(x)=\min \left\{M_{A}(x), M_{A}(1)\right\}=\min \left\{M_{A}(x), M_{A}(x \rightarrow y)\right\} \leq M_{A}(y), \\
& \tilde{B}_{A}(x)=\operatorname{rmin}\left\{\tilde{B}_{A}(x), \tilde{B}_{A}(1)\right\}=\operatorname{rmin}\left\{\tilde{B}_{A}(x), \tilde{B}_{A}(x \rightarrow y)\right\} \preceq \tilde{B}_{A}(y), \\
& J_{A}(x)=\max \left\{J_{A}(x), J_{A}(1)\right\}=\max \left\{J_{A}(x), J_{A}(x \rightarrow y)\right\} \geq J_{A}(y),
\end{aligned}
$$

and so for all $x, y \in X, M_{A}(y) \geq M_{A}(x), \tilde{B}_{A}(y) \succeq \tilde{B}_{A}(x)$ and $J_{A}(y) \leq J_{A}(x)$. By (3.7) and by Proposition 2.2 (ix), we have $M_{A}(x \sim y) \leq M_{A}(x \rightarrow y), \tilde{B}_{A}(x \sim y) \preceq \tilde{B}_{A}(x \rightarrow y)$ and $J_{A}(x \sim y) \geq J_{A}(x \rightarrow y)$, and so

$$
\begin{aligned}
& \min \left\{M_{A}(x), M_{A}(x \sim y)\right\} \leq \min \left\{M_{A}(x), M_{A}(x \rightarrow y)\right\} \leq M_{A}(y), \\
& \operatorname{rmin}\left\{\tilde{B}_{A}(x), \tilde{B}_{A}(x \sim y)\right\} \preceq \operatorname{rmin}\left\{\tilde{B}_{A}(x), \tilde{B}_{A}(x \rightarrow y)\right\} \preceq \tilde{B}_{A}(y), \\
& \max \left\{J_{A}(x), J_{A}(x \sim y)\right\} \geq \max \left\{J_{A}(x), J_{A}(x \rightarrow y)\right\} \geq J_{A}(y) .
\end{aligned}
$$

Therefore, $\mathcal{A}=\left(M_{A}, \tilde{B}_{A}, J_{A}\right)$ is an MBJ-neutrosophic filter of $X$.

In the following example we show that every MBJ-neutrosophic filter in $X$ is not an MBJneutrosophic sub-algebra, in general.

Example 3.11. Let $X$ be the equality algebra as Example 3.2 and $\mathcal{A}=\left(M_{A}, \tilde{B}_{A}, J_{A}\right)$ be an MBJ-neutrosophic filter of $X$ as Example 3.9. It is clear that $\mathcal{A}=\left(M_{A}, \tilde{B}_{A}, J_{A}\right)$ is not an $M B J$-neutrosophic sub-algebra of $X$, because

$$
[0.3,0.4]=\tilde{B}_{A}(0)=\tilde{B}_{A}(a \sim b) \nsucceq \operatorname{rmin}\left\{\tilde{B}_{A}(a), \tilde{B}_{A}(b)\right\}=\tilde{B}_{A}(a)=[0.4,0.5] .
$$

Theorem 3.12. In a linearly order equality algebra, every MBJ-neutrosophic filter is an $M B J-$ neutrosophic sub-algebra. 
Proof. Let $\mathcal{A}=\left(M_{A}, \tilde{B}_{A}, J_{A}\right)$ be an MBJ-neutrosophic filter of $X$. Since for all $x, y \in X$, we have $y \leq x \rightarrow y$, then by $(3.6), M_{A}(y) \leq M_{A}(x \rightarrow y), \tilde{B}_{A}(y) \preceq \tilde{B}_{A}(x \rightarrow y)$ and $J_{A}(y) \geq J_{A}(x \rightarrow y)$. It follows from the assumption that for all $x, y \in X$

$$
\begin{aligned}
& M_{A}(x \rightarrow y) \geq M_{A}(y) \geq \min \left\{M_{A}(x), M_{A}(x \rightarrow y)\right\} \geq \min \left\{M_{A}(x), M_{A}(y)\right\}, \\
& \tilde{B}_{A}(x \rightarrow y) \succeq \tilde{B}_{A}(y) \succeq \operatorname{rmin}\left\{\tilde{B}_{A}(x), \tilde{B}_{A}(x \rightarrow y)\right\} \succeq \operatorname{rmin}\left\{\tilde{B}_{A}(x), \tilde{B}_{A}(y)\right\}, \\
& J_{A}(x \rightarrow y) \leq J_{A}(y) \leq \max \left\{J_{A}(x), J_{A}(x \rightarrow y)\right\}=\leq \max \left\{J_{A}(x), J_{A}(y)\right\} .
\end{aligned}
$$

Hence by Theorem 3.7, $\mathcal{A}=\left(M_{A}, \tilde{B}_{A}, J_{A}\right)$ is an MBJ-neutrosophic sub-algebra of $X$.

Theorem 3.13. An MBJ-neutrosophic set $\mathcal{A}=\left(M_{A}, \tilde{B}_{A}, J_{A}\right)$ in $X$ is an MBJ-neutrosophic filter of $X$ if and only if it satisfies:

$$
(\forall x, y, z \in X)\left(x \leq y \rightarrow z \Rightarrow\left\{\begin{array}{l}
M_{A}(z) \geq \min \left\{M_{A}(x), M_{A}(y)\right\} \\
\tilde{B}_{A}(z) \succeq \operatorname{rmin}\left\{\tilde{B}_{A}(x), \tilde{B}_{A}(y)\right\} \\
J_{A}(z) \leq \max \left\{J_{A}(x), J_{A}(y)\right\}
\end{array}\right) .\right.
$$

Proof. Suppose $\mathcal{A}$ is an MBJ-neutrosophic filter of $X$. Let $x, y, z \in X$ such that $x \leq y \rightarrow z$. Then $M_{A}(x) \leq M_{A}(y \rightarrow z), \tilde{B}_{A}(x) \preceq \tilde{B}_{A}(y \rightarrow z)$ and $J_{A}(x) \geq J_{A}(y \rightarrow z)$. Thus

$$
\begin{aligned}
& \min \left\{M_{A}(y), M_{A}(x)\right\} \leq \min \left\{M_{A}(y), M_{A}(y \rightarrow z)\right\} \leq M_{A}(z), \\
& \operatorname{rmin}\left\{\tilde{B}_{A}(y), \tilde{B}_{A}(x)\right\} \preceq \operatorname{rmin}\left\{\tilde{B}_{A}(y), \tilde{B}_{A}(y \rightarrow z)\right\} \preceq \tilde{B}_{A}(z), \\
& \max \left\{J_{A}(y), J_{A}(x)\right\} \geq \max \left\{J_{A}(y), J_{A}(y \rightarrow z)\right\} \geq J_{A}(z) .
\end{aligned}
$$

Conversly, suppose that $\mathcal{A}$ satisfies in (3.8). Since $x \leq 1$ and for any $x \in X, 1=x \rightarrow 1$, we have $x \leq x \rightarrow 1$. Then by 3.8 we have,

$$
\begin{aligned}
& M_{A}(1) \geq \min \left\{M_{A}(x), M_{A}(x)\right\}=M_{A}(x), \\
& \tilde{B}_{A}(1) \succeq \operatorname{rmin}\left\{\tilde{B}_{A}(x), \tilde{B}_{A}(x)\right\}=\tilde{B}_{A}(x), \\
& J_{A}(1) \leq \max \left\{J_{A}(x), J_{A}(x)\right\}=J_{A}(x)
\end{aligned}
$$

Since for any $x, y \in X, x \rightarrow y \leq x \rightarrow y$, we have

$$
\begin{aligned}
& \min \left\{M_{A}(x), M_{A}(x \rightarrow y)\right\} \leq M_{A}(y), \\
& \operatorname{rmin}\left\{\tilde{B}_{A}(x), \tilde{B}_{A}(x \rightarrow y)\right\} \preceq \tilde{B}_{A}(y), \\
& \max \left\{J_{A}(x), J_{A}(x \rightarrow y)\right\} \geq J_{A}(y) .
\end{aligned}
$$

Hence by Theorem $3.10, \mathcal{A}$ is an MBJ-neutrosophic filter of $X$.

Proposition 3.14. Let $X$ be a positive implicative equality algebra and $\mathcal{A}=\left(M_{A}, \tilde{B}_{A}, J_{A}\right)$ be an $M B J$-neutrosophic set of $X$. Then for any $x, y, z \in X$, the following statements are equivalent: (i) $\mathcal{A}=\left(M_{A}, \tilde{B}_{A}, J_{A}\right)$ is an MBJ-neutrosophic filter of $X$,

$$
\begin{aligned}
& \min \left\{M_{A}(x \rightarrow(y \rightarrow z)), M_{A}(x \rightarrow y)\right\} \leq M_{A}(x \rightarrow z), \\
& \operatorname{rmin}\left\{\tilde{B}_{A}(x \rightarrow(y \rightarrow z)), \tilde{B}_{A}(x \rightarrow y)\right\} \preceq \tilde{B}_{A}(x \rightarrow z), \\
& \max \left\{J_{A}(x \rightarrow(y \rightarrow z)), J_{A}(x \rightarrow y)\right\} \geq J_{A}(x \rightarrow z),
\end{aligned}
$$

$$
\begin{aligned}
& \min \left\{M_{A}(z \rightarrow(y \rightarrow(y \rightarrow x))), M_{A}(z)\right\} \leq M_{A}(y \rightarrow x), \\
& \operatorname{rmin}\left\{\tilde{B}_{A}(z \rightarrow(y \rightarrow(y \rightarrow x))), \tilde{B}_{A}(z)\right\} \preceq \tilde{B}_{A}(y \rightarrow x), \\
& \max \left\{J_{A}(z \rightarrow(y \rightarrow(y \rightarrow x))), J_{A}(z)\right\} \geq J_{A}(y \rightarrow x) .
\end{aligned}
$$


Proof. $\left(i \Rightarrow\right.$ iii) Let $\mathcal{A}=\left(M_{A}, \tilde{B}_{A}, J_{A}\right)$ be an MBJ-neutrosophic filter of $X$. Since $X$ is a positive implicative equality algebra, we have

$$
\begin{aligned}
& \min \left\{M_{A}(z \rightarrow(y \rightarrow(y \rightarrow x))), M_{A}(z)\right\} \leq M_{A}(y \rightarrow(y \rightarrow x))=M_{A}(y \rightarrow x), \\
& \operatorname{rmin}\left\{\tilde{B}_{A}(z \rightarrow(y \rightarrow(y \rightarrow x))), \tilde{B}_{A}(z)\right\} \preceq \tilde{B}_{A}(y \rightarrow(y \rightarrow x))=\tilde{B}_{A}(y \rightarrow x), \\
& \max \left\{J_{A}(z \rightarrow(y \rightarrow(y \rightarrow x))), J_{A}(z)\right\} \geq J_{A}(y \rightarrow(y \rightarrow x))=J_{A}(y \rightarrow x) .
\end{aligned}
$$

$(i i i \Rightarrow i i)$ Since $X$ is a positive implicative equality algebra, we have

$$
\begin{aligned}
& \min \left\{M_{A}(x \rightarrow(y \rightarrow z)), M_{A}(x \rightarrow y)\right\}=\min \left\{M_{A}((x \rightarrow y) \rightarrow(x \rightarrow z)), M_{A}(x \rightarrow y)\right\} \\
& =\min \left\{M_{A}\left((x \rightarrow y) \rightarrow(x \rightarrow(x \rightarrow z)), M_{A}(x \rightarrow y)\right\} \leq M_{A}(x \rightarrow z),\right. \\
& \operatorname{rmin}\left\{\tilde{B}_{A}(x \rightarrow(y \rightarrow z)), \tilde{B}_{A}(x \rightarrow y)\right\}=\min \left\{\tilde{B}_{A}((x \rightarrow y) \rightarrow(x \rightarrow z)), \tilde{B}_{A}(x \rightarrow y)\right\} \\
& =\min \left\{\tilde{B}_{A}\left((x \rightarrow y) \rightarrow(x \rightarrow(x \rightarrow z)), \tilde{B}_{A}(x \rightarrow y)\right\} \preceq \tilde{B}_{A}(x \rightarrow z),\right. \\
& \max \left\{J_{A}(x \rightarrow(y \rightarrow z)), J_{A}(x \rightarrow y)\right\}=\max \left\{J_{A}((x \rightarrow y) \rightarrow(x \rightarrow z)), J_{A}(x \rightarrow y)\right\} \\
& =\max \left\{J_{A}\left((x \rightarrow y) \rightarrow(x \rightarrow(x \rightarrow z)), J_{A}(x \rightarrow y)\right\} \geq J_{A}(x \rightarrow z) .\right.
\end{aligned}
$$

$(i i \Rightarrow i)$ It is enough to let $x=1$.

Proposition 3.15. Let $\mathcal{A}$ be an MBJ-neutrosophic filter of $X$. Then for any $x, y, z, s, t \in X$ the following statements hold:

(1) If $M_{A}(x \rightarrow y)=M_{A}(1)$, then $M_{A}(x) \leq M_{A}(y)$.

(2) If $\tilde{B}_{A}(x \rightarrow y)=\tilde{B}_{A}(1)$, then $\tilde{B}_{A}(x) \preceq \tilde{B}_{A}(y)$.

(3) If $J_{A}(x \rightarrow y)=J_{A}(1)$, then $J_{A}(x) \geq J_{A}(y)$.

(4) $\min \left\{M_{A}(x), M_{A}(x \rightarrow y)\right\} \leq M_{A}(x \wedge y)$,

(5) $\operatorname{rmin}\left\{\tilde{B}_{A}(x), \tilde{B}_{A}(x \rightarrow y)\right\} \preceq \tilde{B}_{A}(x \wedge y)$,

(6) $\max \left\{J_{A}(x), J_{A}(x \rightarrow y)\right\} \geq J_{A}(x \wedge y)$,

(7) $\min \left\{M_{A}(x), M_{A}(y)\right\}=M_{A}(x \wedge y)$,

(8) $\operatorname{rmin}\left\{\tilde{B}_{A}(x), \tilde{B}_{A}(y)\right\}=\tilde{B}_{A}(x \wedge y)$,

(9) $\max \left\{J_{A}(x), J_{A}(y)\right\}=J_{A}(x \wedge y)$,

(10) $\min \left\{M_{A}(x \sim y), M_{A}(y \sim z)\right\} \leq M_{A}(x \sim z)$,

(11) $\operatorname{rmin}\left\{\tilde{B}_{A}(x \sim y), \tilde{B}_{A}(y \sim z)\right\} \preceq \tilde{B}_{A}(x \sim z)$,

(12) $\max \left\{J_{A}(x \sim y), J_{A}(y \sim z)\right\} \geq J_{A}(x \sim z)$,

(13) $\min \left\{M_{A}(x \rightarrow y), M_{A}(y \rightarrow z)\right\} \leq M_{A}(x \rightarrow z)$,

(14) $\operatorname{rmin}\left\{\tilde{B}_{A}(x \rightarrow y), \tilde{B}_{A}(y \rightarrow z)\right\} \preceq \tilde{B}_{A}(x \rightarrow z)$,

(15) $\max \left\{J_{A}(x \rightarrow y), J_{A}(y \rightarrow z)\right\} \geq J_{A}(x \rightarrow z)$,

(16) If $y \leq x$, then $M_{A}(y)=\min \left\{M_{A}(x), M_{A}(x \rightarrow y)\right\}$. 
(17) If $y \leq x$, then $\tilde{B}_{A}(y)=\operatorname{rmin}\left\{\tilde{B}_{A}(x), \tilde{B}_{A}(x \rightarrow y)\right\}$.

(18) If $y \leq x$, then $J_{A}(y)=\max \left\{J_{A}(x), J_{A}(x \rightarrow y)\right\}$.

Proof. (1), (2) and (3) by Theorem 3.10 and assumptions $M_{A}(x \rightarrow y)=M_{A}(1), \tilde{B}_{A}(x \rightarrow y)=$ $\tilde{B}_{A}(1)$ and $J_{A}(x \rightarrow y)=J_{A}(1)$, we have

$$
\begin{aligned}
& M_{A}(x)=\min \left\{M_{A}(x), M_{A}(1)\right\}=\min \left\{M_{A}(x), M_{A}(x \rightarrow y)\right\} \leq M_{A}(y), \\
& \tilde{B}_{A}(x)=\operatorname{rmin}\left\{\tilde{B}_{A}(x), \tilde{B}_{A}(1)\right\}=\operatorname{rmin}\left\{\tilde{B}_{A}(x), \tilde{B}_{A}(x \rightarrow y)\right\} \preceq \tilde{B}_{A}(y), \\
& J_{A}(x)=\max \left\{J_{A}(x), J_{A}(1)\right\}=\max \left\{J_{A}(x), J_{A}(x \rightarrow y)\right\} \geq J_{A}(y) .
\end{aligned}
$$

(4), (5) and (6) by Definition 3.8, we have

$$
\begin{aligned}
& \min \left\{M_{A}(x), M_{A}(x \rightarrow y)\right\}=\min \left\{M_{A}(x), M_{A}(x \sim(x \wedge y))\right\} \leq M_{A}(x \wedge y), \\
& \operatorname{rmin}\left\{\tilde{B}_{A}(x), \tilde{B}_{A}(x \rightarrow y)\right\}=\operatorname{rmin}\left\{\tilde{B}_{A}(x), \tilde{B}_{A}(x \sim(x \wedge y))\right\} \preceq \tilde{B}_{A}(x \wedge y), \\
& \max \left\{J_{A}(x), J_{A}(x \rightarrow y)\right\}=\max \left\{J_{A}(x), J_{A}(x \sim(x \wedge y))\right\} \geq J_{A}(x \wedge y) .
\end{aligned}
$$

(7), (8) and (9) this is clear that $M_{A}(x \wedge y) \leq \min \left\{M_{A}(x), M_{A}(y)\right\}, \tilde{B}_{A}(x \wedge y) \preceq \operatorname{rmin}\left\{\tilde{B}_{A}(x), \tilde{B}_{A}(y)\right\}$ and $J_{A}(x \wedge y) \geq \max \left\{J_{A}(x), J_{A}(y)\right\}$. Conversly, by Proposition 2.2(v) and items (4),(5) and (6), we obtain

$$
\begin{aligned}
& \min \left\{M_{A}(x), M_{A}(y)\right\} \leq \min \left\{M_{A}(x), M_{A}(x \rightarrow y)\right\} \leq M_{A}(x \wedge y), \\
& \operatorname{rmin}\left\{\tilde{B}_{A}(x), \tilde{B}_{A}(y)\right\} \preceq \operatorname{rmin}\left\{\tilde{B}_{A}(x), \tilde{B}_{A}(x \rightarrow y)\right\} \preceq \tilde{B}_{A}(x \wedge y), \\
& \max \left\{J_{A}(x), J_{A}(y)\right\} \geq \max \left\{J_{A}(x), J_{A}(x \rightarrow y)\right\} \geq J_{A}(x \wedge y) .
\end{aligned}
$$

(10), (11) and (12) since $x \sim y \leq(y \sim z) \sim(x \sim z)$, by Definition 2.1 (E7) and Definition 3.8, we get

$$
\begin{aligned}
& \min \left\{M_{A}(x \sim y), M_{A}(y \sim z)\right\} \leq \min \left\{M_{A}((y \sim z) \sim(x \sim z)), M_{A}(y \sim z)\right\} \leq M_{A}(x \sim z), \\
& \operatorname{rmin}\left\{\tilde{B}_{A}(x \sim y), \tilde{B}_{A}(y \sim z)\right\} \preceq \operatorname{rmin}\left\{\tilde{B}_{A}((y \sim z) \sim(x \sim z)), \tilde{B}_{A}(y \sim z)\right\} \preceq \tilde{B}_{A}(x \sim z),
\end{aligned}
$$$$
\max \left\{J_{A}(x \sim y), J_{A}(y \sim z)\right\} \geq \max \left\{J_{A}((y \sim z) \sim(x \sim z)), J_{A}(y \sim z)\right\} \geq J_{A}(x \sim z) .
$$

(13), (14) and (15) by Proposition 2.2(vii), we have $x \rightarrow y \leq(y \rightarrow z) \rightarrow(x \rightarrow z)$, then by Definition 3.8 and Theorem 3.10 we get

$$
\begin{aligned}
& \min \left\{M_{A}(x \rightarrow y), M_{A}(y \rightarrow z)\right\} \leq \min \left\{M_{A}((y \rightarrow z) \rightarrow(x \rightarrow z)), M_{A}(y \rightarrow z)\right\} \leq M_{A}(x \rightarrow z), \\
& \operatorname{rmin}\left\{\tilde{B}_{A}(x \rightarrow y), \tilde{B}_{A}(y \rightarrow z)\right\} \preceq \operatorname{rmin}\left\{\tilde{B}_{A}((y \rightarrow z) \rightarrow(x \rightarrow z)), \tilde{B}_{A}(y \rightarrow z)\right\} \preceq \tilde{B}_{A}(x \rightarrow z), \\
& \max \left\{J_{A}(x \rightarrow y), J_{A}(y \rightarrow z)\right\} \geq \max \left\{J_{A}((y \rightarrow z) \rightarrow(x \rightarrow z)), J_{A}(y \rightarrow z)\right\} \geq J_{A}(x \rightarrow z) .
\end{aligned}
$$

(16), (17) and (18) since $y \leq x, y \leq x \rightarrow y$ and $\mathcal{A}$ is an MBJ-neutrosophic filter of $X$, we have

$$
\begin{aligned}
& M_{A}(y) \leq \min \left\{M_{A}(x \rightarrow y), M_{A}(x)\right\} \leq M_{A}(y), \\
& \tilde{B}_{A}(y) \preceq \operatorname{rmin}\left\{\tilde{B}_{A}(x \rightarrow y), \tilde{B}_{A}(x)\right\} \preceq \tilde{B}_{A}(y), \\
& J_{A}(y) \geq \max \left\{J_{A}(x \rightarrow y), J_{A}(x)\right\} \geq J_{A}(y) .
\end{aligned}
$$

Proposition 3.16. Let $\left\{F_{i}\right\}_{i \in \Lambda}$ be a family of MBJ-neutrosophic filters of $X$. Then infinite intersection $\left(\bigwedge_{i \in \Lambda}\right)$ of $F_{i}$ is an MBJ-neutrosophic filter of $X$. 
Proof. Suppose that for any $x, y, z \in X, x \leq y \rightarrow z$. Since $F_{i}$ are an MBJ-neutrosophic filters of $X$, by Theorem 3.13 for all $x, y, z \in X$, we have

$$
\begin{aligned}
& M_{A}(z) \geq \min \left\{M_{A}(x), M_{A}(y)\right\}, \\
& \tilde{B}_{A}(z) \succeq \operatorname{rmin}\left\{\tilde{B}_{A}(x), \tilde{B}_{A}(y)\right\}, \\
& J_{A}(z) \leq \max \left\{J_{A}(x), J_{A}(y)\right\},
\end{aligned}
$$

Thus,

$$
\begin{aligned}
\bigwedge_{i \in \Lambda} M_{A}^{i}(z) & =\inf _{i \in \Lambda} M_{A}^{i}(z) \geq \inf _{i \in \Lambda}\left(\min \left\{M_{A}^{i}(x), M_{A}^{i}(y)\right\}\right)=\min \left\{\inf _{i \in \Lambda} M_{A}^{i}(x), \inf _{i \in \Lambda} M_{A}^{i}(y)\right\} \\
& =\min \left\{\bigwedge_{i \in \Lambda} M_{A}^{i}(x), \bigwedge_{i \in \Lambda} M_{A}^{i}(y)\right\} \\
\bigwedge_{i \in \Lambda} \tilde{B}_{A}^{i}(z)= & \operatorname{rinf}_{i \in \Lambda} \tilde{B}_{A}^{i}(z) \succeq \operatorname{rinf}_{i \in \Lambda}\left(\operatorname{rmin}\left\{\tilde{B}_{A}^{i}(x), \tilde{B}_{A}^{i}(y)\right\}\right)=\operatorname{rmin}\left\{\operatorname{rinf}_{i \in \Lambda} \tilde{B}_{A}^{i}(x), \operatorname{rinf}_{i \in \Lambda} \tilde{B}_{A}^{i}(y)\right\} \\
= & \min \left\{\bigwedge_{i \in \Lambda} \tilde{B}_{A}^{i}(x), \bigwedge_{i \in \Lambda} \tilde{B}_{A}^{i}(y)\right\}, \\
\bigwedge_{i \in \Lambda} J_{A}^{i}(z) & =\inf _{i \in \Lambda} J_{A}^{i}(z) \leq \inf _{i \in \Lambda}\left(\max \left\{J_{A}^{i}(x), J_{A}^{i}(y)\right\}\right)=\max \left\{\inf _{i \in \Lambda} J_{A}^{i}(x), \inf _{i \in \Lambda} J_{A}^{i}(y)\right\} \\
& =\max \left\{\bigwedge_{i \in \Lambda} J_{A}^{i}(x), \bigwedge_{i \in \Lambda} J_{A}^{i}(y)\right\} .
\end{aligned}
$$

Therefore, by Theorem 3.13, infinite intersection of $F_{i}$ is an MBJ-neutrosophic filter of $X$.

Let $X$ and $Y$ be two equality algebras. $f: X \rightarrow Y$ is called an equality homomorphism if for any $x, y \in X$, we have

$$
f(x \sim y)=f(x) \sim f(y), \text { and } f(x \wedge y)=f(x) \wedge f(y) .
$$

It is clear that $f(x \rightarrow y)=f(x) \rightarrow f(y)$ and if $X$ and $Y$ are bounded, then $f(0)=0$.

Notation. Let $X$ and $Y$ be two equality algebras and $f: X \rightarrow Y$ be an equality homomorphism. For any MBJ-neutrosophic set $\mathcal{A}=\left(M_{A}, \tilde{B}_{A}, J_{A}\right)$ in $Y$, we define a new MBJ-neutrosophic set $\mathcal{A}^{f}=\left(M_{A}^{f}, \tilde{B}_{A}^{f}, J_{A}^{f}\right)$ in $X$ by $M_{A}^{f}(x)=M_{A}(f(x)), \tilde{B}_{A}^{f}(x)=\tilde{B}_{A}(f(x))$ and $J_{A}^{f}(x)=J_{A}(f(x))$, for any $x \in X$.

Theorem 3.17. Let $f: X \rightarrow Y$ be an equality homomorphism.

(i) If $\mathcal{A}=\left(M_{A}, \tilde{B}_{A}, J_{A}\right)$ is an MBJ-neutrosophic filter of $Y$, then $\mathcal{A}^{f}=\left(M_{A}^{f}, \tilde{B}_{A}^{f}\right.$, $\left.J_{A}^{f}\right)$ is an MBJ-neutrosophic filter of $X$.

(ii) If $f$ is an equality epimorphism and $\mathcal{A}^{f}=\left(M_{A}^{f}, \tilde{B}_{A}^{f}, J_{A}^{f}\right)$ is an MBJ-neutrosophic filter of $X$, then $\mathcal{A}=\left(M_{A}, \tilde{B}_{A}, J_{A}\right)$ is an MBJ-neutrosophic filter of $Y$.

Proof. (i) Let $x, y \in X$. Since $f(x) \leq 1=f(1)$ and $\mathcal{A}=\left(M_{A}, \tilde{B}_{A}, J_{A}\right)$ is an MBJ-neutrosophic filter of $Y$, we have

$$
\begin{aligned}
& M_{A}^{f}(x)=M_{A}(f(x)) \leq M_{A}(f(1))=M_{A}^{f}(1), \\
& \tilde{B}_{A}^{f}(x)=\tilde{B}_{A}(f(x)) \preceq \tilde{B}_{A}(f(1))=\tilde{B}_{A}^{f}(1), \\
& J_{A}^{f}(x)=J_{A}(f(x)) \geq J_{A}(f(1))=J_{A}^{f}(1) .
\end{aligned}
$$


Moreover, since $f$ is an equality homomotphism, we get

$$
\begin{aligned}
\min \left\{M_{A}^{f}(x), M_{A}^{f}(x \rightarrow y)\right\} & =\min \left\{M_{A}(f(x \rightarrow y)), M_{A}(f(x))\right\}=\min \left\{M_{A}(f(x) \rightarrow f(y)), M_{A}(f(x))\right\} \\
& \leq M_{A}(f(y))=M_{A}^{f}(y), \\
\min \left\{\tilde{B}_{A}^{f}(x), \tilde{B}_{A}^{f}(x \rightarrow y)\right\} & =\min \left\{\tilde{B}_{A}(f(x \rightarrow y)), \tilde{B}_{A}(f(x))\right\}=\min \left\{\tilde{B}_{A}(f(x) \rightarrow f(y)), \tilde{B}_{A}(f(x))\right\} \\
& \preceq \tilde{B}_{A}(f(y))=\tilde{B}_{A}^{f}(y), \\
\max \left\{M_{A}^{f}(x), M_{A}^{f}(x \rightarrow y)\right\} & =\max \left\{M_{A}(f(x \rightarrow y)), M_{A}(f(x))\right\}=\max \left\{M_{A}(f(x) \rightarrow f(y)), M_{A}(f(x))\right\} \\
& \geq M_{A}(f(y))=M_{A}^{f}(y),
\end{aligned}
$$

Therefore $\mathcal{A}^{f}=\left(M_{A}^{f}, \tilde{B}_{A}^{f}, J_{A}^{f}\right)$ is an MBJ-neutrosophic filter of $X$.

(ii) Let $y \in Y$. Since $f$ is an equality epimorphism and $\mathcal{A}^{f}=\left(M_{A}^{f}, \tilde{B}_{A}^{f}, J_{A}^{f}\right)$ is an MBJ-neutrosophic filter of $X$, there exists $x \in X$ such that $f(x)=y$ and

$$
\begin{aligned}
& M_{A}(y)=M_{A}(f(x))=M_{A}^{f}(x) \leq M_{A}^{f}(1)=M_{A}(f(1))=M_{A}(1), \\
& \tilde{B}_{A}(y)=\tilde{B}_{A}(f(x))=\tilde{B}_{A}^{f}(x) \preceq \tilde{B}_{A}^{f}(1)=\tilde{B}_{A}(f(1))=\tilde{B}_{A}(1), \\
& J_{A}(y)=J_{A}(f(x))=J_{A}^{f}(x) \geq J_{A}^{f}(1)=J_{A}(f(1))=J_{A}(1) .
\end{aligned}
$$

Now, suppose $x, y \in Y$. Then there exist $a, b \in X$ such that $f(a)=x$ and $f(b)=y$. Thus

$$
\begin{aligned}
M_{A}(y) & =M_{A}(f(b))=M_{A}^{f}(b) \geq \min \left\{M_{A}^{f}(a), M_{A}^{f}(a \rightarrow b)\right\}=\min \left\{M_{A}(f(a \rightarrow b)), M_{A}(f(a))\right\} \\
& =\min \left\{M_{A}(f(a) \rightarrow f(b)), M_{A}(f(a))\right\}=\min \left\{M_{A}(x \rightarrow y), M_{A}(x)\right\} . \\
\tilde{B}_{A}(y) & =\tilde{B}_{A}(f(b))=\tilde{B}_{A}^{f}(b) \preceq \min \left\{\tilde{B}_{A}^{f}(a), \tilde{B}_{A}^{f}(a \rightarrow b)\right\}=\min \left\{\tilde{B}_{A}(f(a \rightarrow b)), \tilde{B}_{A}(f(a))\right\} \\
& =\min \left\{\tilde{B}_{A}(f(a) \rightarrow f(b)), \tilde{B}_{A}(f(a))\right\}=\min \left\{\tilde{B}_{A}(x \rightarrow y), \tilde{B}_{A}(x)\right\} . \\
J_{A}(y) & =J_{A}(f(b))=J_{A}^{f}(b) \leq \min \left\{J_{A}^{f}(a), J_{A}^{f}(a \rightarrow b)\right\}=\min \left\{J_{A}(f(a \rightarrow b)), J_{A}(f(a))\right\} \\
& =\min \left\{J_{A}(f(a) \rightarrow f(b)), J_{A}(f(a))\right\}=\min \left\{J_{A}(x \rightarrow y), J_{A}(x)\right\} .
\end{aligned}
$$

Therefore, $\mathcal{A}=\left(M_{A}, \tilde{B}_{A}, J_{A}\right)$ is an MBJ-neutrosophic filter of $Y$.

Define $K(x, y)=\{z \in X \mid x \leq y \rightarrow z\}$ which is called an upper set of $x$ and $y$. It is easy to see that $1, x, y \in K(x, y)$, for any $x, y \in X$.

In the following example we can see that every $K(x, y)$ is not a filter of $X$ in general.

Example 3.18. Let $X=\{0, a, b, c, d, 1\}$ be a set with the following Hasse diagram.

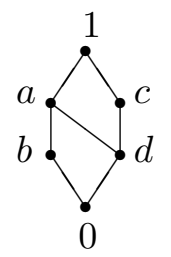

Then $(X, \wedge, 1)$ is a meet semilattice with top element 1 . Define an operation $\sim$ on $X$ by Table 7. 


\begin{tabular}{ccccccc}
\hline$\sim$ & 0 & $a$ & $b$ & $c$ & $d$ & 1 \\
\hline 0 & 1 & $d$ & $c$ & $b$ & $a$ & 0 \\
$a$ & $d$ & 1 & $a$ & $d$ & $c$ & $a$ \\
$b$ & $c$ & $a$ & 1 & 0 & $d$ & $b$ \\
$c$ & $b$ & $d$ & 0 & 1 & $a$ & $c$ \\
$d$ & $a$ & $c$ & $d$ & $a$ & 1 & $d$ \\
1 & 0 & $a$ & $b$ & $c$ & $d$ & 1 \\
\hline
\end{tabular}

Table 7: Cayley table for the binary operation " "

Thus $\mathcal{E}=(X, \wedge, \sim, 1)$ is an equality algebra, and the implication " $\rightarrow$ " is given by Table 8 .

\begin{tabular}{ccccccc}
\hline$\rightarrow$ & 0 & $a$ & $b$ & $c$ & $d$ & 1 \\
\hline 0 & 1 & 1 & 1 & 1 & 1 & 1 \\
$a$ & $d$ & 1 & $a$ & $c$ & $c$ & 1 \\
$b$ & $c$ & 1 & 1 & $c$ & $c$ & 1 \\
$c$ & $b$ & $a$ & $b$ & 1 & $a$ & 1 \\
$d$ & $a$ & 1 & $a$ & 1 & 1 & 1 \\
1 & 0 & $a$ & $b$ & $c$ & $d$ & 1 \\
\hline
\end{tabular}

Table 8: Cayley table for the implication " $\rightarrow$ "

Hence, $K(c, a)=\{a, c, d, 1\}$ is not a filter of $X$, because $d \rightarrow b=a \in K(c, a)$ and $d \in K(c, a)$, but $b \notin K(c, a)$.

Given an MBJ-neutrosophic set $\mathcal{A}=\left(M_{A}, \tilde{B}_{A}, J_{A}\right)$ in $X$, we consider the following sets,

$$
\begin{aligned}
& U\left(M_{A} ; t\right):=\left\{x \in X \mid M_{A}(x) \geq t\right\} \\
& U\left(\tilde{B}_{A} ;\left[\delta_{1}, \delta_{2}\right]\right):=\left\{x \in X \mid \tilde{B}_{A}(x) \succeq\left[\delta_{1}, \delta_{2}\right]\right\} \\
& L\left(J_{A} ; s\right):=\left\{x \in X \mid J_{A}(x) \leq s\right\} .
\end{aligned}
$$

Where $t, s \in[0,1]=I$ and $\left[\delta_{1}, \delta_{2}\right] \in[I]$.

Example 3.19. Let $\mathcal{A}=\left(M_{A}, \tilde{B}_{A}, J_{A}\right)$ be an MBJ-neutrosophic as Example 3.2. Suppose $t=0.5$, $s=0.4$ and $\left[\delta_{1}, \delta_{2}\right]=[0.4,0.5]$. Then it is clear that

$$
\begin{aligned}
& U\left(M_{A} ; 0.5\right):=\left\{x \in X \mid M_{A}(x) \geq 0.5\right\}=\{a, 1\}, \\
& U\left(\tilde{B}_{A} ;[0.4,0.5]\right):=\left\{x \in X \mid \tilde{B}_{A}(x) \succeq[0.4,0.5]\right\}=\{0,1\}, \\
& L\left(J_{A} ; 0.4\right):=\left\{x \in X \mid J_{A}(x) \leq 0.4\right\}=\{1\} .
\end{aligned}
$$

Theorem 3.20. An MBJ-neutrosophic set $\mathcal{A}=\left(M_{A}, \tilde{B}_{A}, J_{A}\right)$ in $X$ is an MBJ-neutrosophic filter of $X$ if and only if for any $t, s \in[0,1]$ and $\left[\delta_{1}, \delta_{2}\right] \in[I]$, the non-empty sets $U\left(M_{A} ; t\right), U\left(\tilde{B}_{A} ;\left[\delta_{1}, \delta_{2}\right]\right)$ and $L\left(J_{A} ; s\right)$ are filters of $X$.

Proof. Suppose $\mathcal{A}=\left(M_{A}, \tilde{B}_{A}, J_{A}\right)$ is an MBJ-neutrosophic filter of $X$. Let $t, s \in[0,1]$ and $\left[\delta_{1}, \delta_{2}\right] \in[I]$ such that $U\left(M_{A} ; t\right), U\left(\tilde{B}_{A} ;\left[\delta_{1}, \delta_{2}\right]\right)$ and $L\left(J_{A} ; s\right)$ are non-empty sets. Obviously, 
$1 \in U\left(M_{A} ; t\right), 1 \in U\left(\tilde{B}_{A} ;\left[\delta_{1}, \delta_{2}\right]\right)$ and $1 \in L\left(J_{A} ; s\right)$. For any $x, y, a, b, u, v \in X$, if $x, x \rightarrow y \in$ $U\left(M_{A} ; t\right), a, a \rightarrow b \in U\left(\tilde{B}_{A} ;\left[\delta_{1}, \delta_{2}\right]\right)$ and $u, u \rightarrow v \in L\left(J_{A} ; s\right)$, then

$$
\begin{aligned}
& M_{A}(y) \geq \min \left\{M_{A}(x), M_{A}(x \rightarrow y)\right\} \geq \min \{t, t\}=t, \\
& \tilde{B}_{A}(b) \succeq \operatorname{rmin}\left\{\tilde{B}_{A}(a), \tilde{B}_{A}(a \rightarrow b)\right\} \succeq \operatorname{rmin}\left\{\left[\delta_{1}, \delta_{2}\right],\left[\delta_{1}, \delta_{2}\right]\right\}=\left[\delta_{1}, \delta_{2}\right], \\
& J_{A}(v) \leq \max \left\{J_{A}(u), J_{A}(u \rightarrow v)\right\} \leq \max \{s, s\}=s,
\end{aligned}
$$

and so $y \in U\left(M_{A} ; t\right), b \in U\left(\tilde{B}_{A} ;\left[\delta_{1}, \delta_{2}\right]\right)$ and $v \in L\left(J_{A} ; s\right)$. Therefore, $U\left(M_{A} ; t\right), U\left(\tilde{B}_{A} ;\left[\delta_{1}, \delta_{2}\right]\right)$ and $L\left(J_{A} ; s\right)$ are filters of $X$.

Conversely, suppose that for any $t, s \in[0,1]$ and $\left[\delta_{1}, \delta_{2}\right] \in[I]$, the non-empty sets $U\left(M_{A} ; t\right)$, $L\left(J_{A} ; s\right)$ and $U\left(\tilde{B}_{A} ;\left[\delta_{1}, \delta_{2}\right]\right)$ are filters of $X$. Let $x \in U\left(M_{A} ; t\right) \cap L\left(J_{A} ; s\right) \cap U\left(\tilde{B}_{A} ;\left[\delta_{1}, \delta_{2}\right]\right)$, for some $x \in X$. If $M_{A}(1)<M_{A}(x), \tilde{B}_{A}(1) \prec \tilde{B}_{A}(x)$ and $J_{A}(1)>J_{A}(x)$, then $1 \notin U\left(M_{A} ; t\right), 1 \notin$ $U\left(\tilde{B}_{A} ;\left[\delta_{1}, \delta_{2}\right]\right)$ and $1 \notin L\left(J_{A} ; s\right)$, which is a contradiction. Hence $M_{A}(x) \leq M_{A}(1), \tilde{B}_{A}(x) \preceq$ $\tilde{B}_{A}(1), J_{A}(x) \geq J_{A}(1)$ for all $x \in X$. Suppose for some $x_{0}, y_{0} \in X, x_{0}, x_{0} \rightarrow y_{0} \in U\left(M_{A} ; t_{0}\right)$ but $y_{0} \notin U\left(M_{A} ; t_{0}\right)$ for $t_{0}:=\min \left\{M_{A}\left(x_{0}\right), M_{A}\left(x_{0} \rightarrow y_{0}\right)\right\}$. This is a contradiction and thus $M_{A}(y) \geq \min \left\{M_{A}(x), M_{A}(x \rightarrow y)\right\}$ for all $x, y \in X$. Similarly, we can show that for all $x, y \in X$, $J_{A}(y) \leq \max \left\{J_{A}(x), J_{A}(x \rightarrow y)\right\}$. Suppose for some $x_{0}, y_{0} \in X, \tilde{B}_{A}\left(y_{0}\right) \prec \operatorname{rmin}\left\{\tilde{B}_{A}\left(x_{0}\right), \tilde{B}_{A}\left(x_{0} \rightarrow\right.\right.$ $\left.\left.y_{0}\right)\right\}$. Let $\tilde{B}_{A}\left(x_{0} \rightarrow y_{0}\right)=\left[\eta_{1}, \eta_{2}\right], \tilde{B}_{A}\left(x_{0}\right)=\left[\eta_{3}, \eta_{4}\right]$ and $\tilde{B}_{A}\left(y_{0}\right)=\left[\delta_{1}, \delta_{2}\right]$. Then

$$
\left[\delta_{1}, \delta_{2}\right] \prec \operatorname{rmin}\left\{\left[\eta_{1}, \eta_{2}\right],\left[\eta_{3}, \eta_{4}\right]\right\}=\left[\min \left\{\eta_{1}, \eta_{3}\right\}, \min \left\{\eta_{2}, \eta_{4}\right\}\right],
$$

and so $\delta_{1}<\min \left\{\eta_{1}, \eta_{3}\right\}$ and $\delta_{2}<\min \left\{\eta_{2}, \eta_{4}\right\}$. Taking

$$
\left[\gamma_{1}, \gamma_{2}\right]:=\frac{1}{2}\left(\tilde{B}_{A}\left(y_{0}\right)+\operatorname{rmin}\left\{\tilde{B}_{A}\left(x_{0} \rightarrow y_{0}\right), \tilde{B}_{A}\left(x_{0}\right)\right\}\right),
$$

implies that

$$
\begin{aligned}
{\left[\gamma_{1}, \gamma_{2}\right] } & =\frac{1}{2}\left(\left[\delta_{1}, \delta_{2}\right]+\operatorname{rmin}\left\{\left[\eta_{1}, \eta_{2}\right],\left[\eta_{3}, \eta_{4}\right]\right\}\right)=\frac{1}{2}\left(\left[\delta_{1}, \delta_{2}\right]+\left[\min \left\{\eta_{1}, \eta_{3}\right\}, \min \left\{\eta_{2}, \eta_{4}\right\}\right]\right) \\
& =\left[\frac{1}{2}\left(\delta_{1}+\min \left\{\eta_{1}, \eta_{3}\right\}\right), \frac{1}{2}\left(\delta_{2}+\min \left\{\eta_{2}, \eta_{4}\right\}\right)\right] .
\end{aligned}
$$

It follows that

$$
\min \left\{\eta_{1}, \eta_{3}\right\}>\gamma_{1}=\frac{1}{2}\left(\delta_{1}+\min \left\{\eta_{1}, \eta_{3}\right\}\right)>\delta_{1},
$$

and

$$
\min \left\{\eta_{2}, \eta_{4}\right\}>\gamma_{2}=\frac{1}{2}\left(\delta_{2}+\min \left\{\eta_{2}, \eta_{4}\right\}\right)>\delta_{2}
$$

Hence

$$
\left[\min \left\{\eta_{1}, \eta_{3}\right\}, \min \left\{\eta_{2}, \eta_{4}\right\}\right] \succ\left[\gamma_{1}, \gamma_{2}\right] \succ\left[\delta_{1}, \delta_{2}\right]=\tilde{B}_{A}\left(y_{0}\right),
$$

and so $y_{0} \notin U\left(\tilde{B}_{A} ;\left[\gamma_{1}, \gamma_{2}\right]\right)$. On the other hand,

$$
\tilde{B}_{A}\left(x_{0} \rightarrow y_{0}\right)=\left[\eta_{1}, \eta_{2}\right] \succeq\left[\min \left\{\eta_{1}, \eta_{3}\right\}, \min \left\{\eta_{2}, \eta_{4}\right\}\right] \succ\left[\gamma_{1}, \gamma_{2}\right],
$$

and

$$
\tilde{B}_{A}\left(x_{0}\right)=\left[\eta_{3}, \eta_{4}\right] \succeq\left[\min \left\{\eta_{1}, \eta_{3}\right\}, \min \left\{\eta_{2}, \eta_{4}\right\}\right] \succ\left[\gamma_{1}, \gamma_{2}\right],
$$

so $x_{0} \rightarrow y_{0}, x_{0} \in U\left(\tilde{B}_{A} ;\left[\gamma_{1}, \gamma_{2}\right]\right)$, which is a contradiction and so $\tilde{B}_{A}(y) \succeq \operatorname{rmin}\left\{\tilde{B}_{A}(x), \tilde{B}_{A}(x \rightarrow y)\right\}$ for all $x, y \in X$. Therefore, $\mathcal{A}=\left(M_{A}, \tilde{B}_{A}, J_{A}\right)$ is an MBJ-neutrosophic filter of $X$. 
Proposition 3.21. Let $\mathcal{A}=\left(M_{A}, \tilde{B}_{A}, J_{A}\right)$ be an $M B J$-neutrosophic filter of $X$ such that $x, y \in X$, $t, s \in[0,1]$ and $\left[\delta_{1}, \delta_{2}\right] \in[I]$. If $x, y \in U\left(M_{A} ; t\right) \cap U\left(\tilde{B}_{A} ;\left[\delta_{1}, \delta_{2}\right]\right) \cap L\left(J_{A} ; s\right)$, then $K(x, y) \subseteq$ $U\left(M_{A} ; t\right) \cap U\left(\tilde{B}_{A} ;\left[\delta_{1}, \delta_{2}\right]\right) \cap L\left(J_{A} ; s\right)$.

Proof. Let $x, y \in U\left(M_{A} ; t\right) \cap U\left(\tilde{B}_{A} ;\left[\delta_{1}, \delta_{2}\right]\right) \cap L\left(J_{A} ; s\right), t, s \in[0,1],\left[\delta_{1}, \delta_{2}\right] \in[I]$ and $z \in K(x, y)$. Then $x \leq y \rightarrow z$. Since $x, y \in U\left(M_{A} ; t\right) \cap U\left(\tilde{B}_{A} ;\left[\delta_{1}, \delta_{2}\right]\right) \cap L\left(J_{A} ; s\right)$ and $\mathcal{A}$ is an MBJ-neutrosophic filter of $X$, we have $M_{A}(x), M_{A}(y) \geq t$. By Theorem 3.13 , we get $M_{A}(z) \geq \min \left\{M_{A}(x), M_{A}(y)\right\} \geq$ $t$, and so $z \in U\left(M_{A} ; t\right)$. By the similar way, we can see that $z \in U\left(\tilde{B}_{A} ;\left[\delta_{1}, \delta_{2}\right]\right) \cap L\left(J_{A} ; s\right)$. Hence $K(x, y) \subseteq U\left(M_{A} ; t\right) \cap U\left(\tilde{B}_{A} ;\left[\delta_{1}, \delta_{2}\right]\right) \cap L\left(J_{A} ; s\right)$.

Corollary 3.22. Let $\mathcal{A}=\left(M_{A}, \tilde{B}_{A}, J_{A}\right)$ be an MBJ-neutrosophic filter of $X$ such that $t, s \in[0,1]$ and $\left[\delta_{1}, \delta_{2}\right] \in[I]$. If $U\left(M_{A} ; t\right) \cap U\left(\tilde{B}_{A} ;\left[\delta_{1}, \delta_{2}\right]\right) \cap L\left(J_{A} ; s\right) \neq \emptyset$, then

$U\left(M_{A} ; t\right)=\bigcup_{x, y \in U\left(M_{A} ; t\right)} K(x, y), U\left(\tilde{B}_{A} ;\left[\delta_{1}, \delta_{2}\right]\right)=\bigcup_{x, y \in U\left(\tilde{B}_{A} ; t\right)} K(x, y), L\left(J_{A} ; s\right)=\bigcup_{x, y \in U\left(J_{A} ; t\right)} K(x, y)$.

Proof. The proof is straightforward.

Theorem 3.23. Given an filter $F$ of $X$, let $\mathcal{A}=\left(M_{A}, \tilde{B}_{A}, J_{A}\right)$ be an MBJ-neutrosophic set in $X$ that defined by

$$
M_{A}(x)=\left\{\begin{array}{ll}
t & \text { if } x \in F, \\
0 & \text { otherwise, }
\end{array} \quad \tilde{B}_{A}(x)=\left\{\begin{array}{ll}
{\left[\delta_{1}, \delta_{2}\right]} & \text { if } x \in F, \\
{[0,0]} & \text { otherwise, }
\end{array} \quad J_{A}(x)=\left\{\begin{array}{cc}
s & \text { if } x \in F, \\
1 & \text { otherwise },
\end{array}\right.\right.\right.
$$

Then $\mathcal{A}=\left(M_{A}, \tilde{B}_{A}, J_{A}\right)$ is an MBJ-neutrosophic filter of $X$ such that $U\left(M_{A} ; t\right)=U\left(\tilde{B}_{A} ;\left[\delta_{1}, \delta_{2}\right]\right)=$ $L\left(J_{A} ; s\right)=F$.

Proof. Let $x, y \in X$ and $x, x \rightarrow y \in F$. Since $F$ is a filter of $X$, we have $y \in F$ and so

$$
\begin{aligned}
& M_{A}(y)=t=\min \left\{M_{A}(x), M_{A}(x \rightarrow y)\right\}, \\
& \tilde{B}_{A}(y)=\left[\delta_{1}, \delta_{2}\right]=\operatorname{rmin}\left\{\tilde{B}_{A}(x), \tilde{B}_{A}(x \rightarrow y)\right\}, \\
& J_{A}(y)=s=\max \left\{J_{A}(x), J_{A}(x \rightarrow y)\right\} .
\end{aligned}
$$

If $x \rightarrow y \in F$ and $x \notin F$, then $M_{A}(x \rightarrow y)=t, \tilde{B}_{A}(x \rightarrow y)=\left[\delta_{1}, \delta_{2}\right]$ and $J_{A}(x \rightarrow y)=s$. Also, $M_{A}(x)=0, \tilde{B}_{A}(x)=[0,0]$ and $J_{A}(x)=1$. Hence

$$
\begin{aligned}
& M_{A}(y) \geq 0=\min \{t, 0\}=\min \left\{M_{A}(x), M_{A}(x \rightarrow y)\right\}, \\
& \tilde{B}_{A}(y) \succeq[0,0]=\operatorname{rmin}\left\{\left[\delta_{1}, \delta_{2}\right],[0,0]\right\}=\operatorname{rmin}\left\{\tilde{B}_{A}(x), \tilde{B}_{A}(x \rightarrow y)\right\}, \\
& J_{A}(y) \leq 1=\max \{s, 1\}=\max \left\{J_{A}(x), J_{A}(x \rightarrow y)\right\} .
\end{aligned}
$$

If $x \rightarrow y, x \notin F$, then $M_{A}(x)=M_{A}(x \rightarrow y)=0, \tilde{B}_{A}(x)=\tilde{B}_{A}(x \rightarrow y)=[0,0]$ and $J_{A}(x)=$ $J_{A}(x \rightarrow y)=1$. Hence

$$
\begin{aligned}
& M_{A}(y) \geq 0=\min \{0,0\}=\min \left\{M_{A}(x), M_{A}(x \rightarrow y)\right\}, \\
& \tilde{B}_{A}(y) \succeq[0,0]=\operatorname{rmin}\{[0,0],[0,0]\}=\operatorname{rmin}\left\{\tilde{B}_{A}(x), \tilde{B}_{A}(x \rightarrow y)\right\}, \\
& J_{A}(y) \leq 1=\max \{1,1\}=\max \left\{J_{A}(x), J_{A}(x \rightarrow y)\right\} .
\end{aligned}
$$

Since $F$ is a filter of $X$, we have $1 \in F$ and so it is clear that for all $x \in X, M_{A}(x) \leq M_{A}(1), \tilde{B}_{A}(x) \preceq$ $\tilde{B}_{A}(1), J_{A}(x) \geq J_{A}(1)$. Therefore $\mathcal{A}=\left(M_{A}, \tilde{B}_{A}, J_{A}\right)$ is an MBJ-neutrosophic filter of $X$ and we have $U\left(M_{A} ; t\right)=U\left(\tilde{B}_{A} ;\left[\delta_{1}, \delta_{2}\right]\right)=L\left(J_{A} ; s\right)=F$. 
Theorem 3.24. For any non-empty subset $F$ of $X$, let $\mathcal{A}=\left(M_{A}, \tilde{B}_{A}, J_{A}\right)$ be an MBJ-neutrosophic set of $X$ which is given in (3.12). If $\mathcal{A}=\left(M_{A}, \tilde{B}_{A}, J_{A}\right)$ is an MBJ-neutrosophic filter of $X$, then $F$ is a filter of $X$.

Proof. Since for any $x \in U\left(M_{A} ; t\right) \cap L\left(J_{A} ; s\right) \cap U\left(\tilde{B}_{A} ;\left[\delta_{1}, \delta_{2}\right]\right), M_{A}(x) \leq M_{A}(1), \tilde{B}_{A}(x) \preceq \tilde{B}_{A}(1)$, $J_{A}(x) \geq J_{A}(1)$, it is clear that $1 \in F$. Let $x, y \in X$ such that $x \rightarrow y \in F$ and $x \in F$. Then $M_{A}(x)=M_{A}(x \rightarrow y)=t, \tilde{B}_{A}(x)=\tilde{B}_{A}(x \rightarrow y)=\left[\delta_{1}, \delta_{2}\right]$ and $J_{A}(x)=J_{A}(x \rightarrow y)=s$. Thus

$$
\begin{aligned}
& M_{A}(y) \geq \min \left\{M_{A}(x), M_{A}(x \rightarrow y)\right\}=t, \\
& \tilde{B}_{A}(y) \succeq \operatorname{rmin}\left\{\tilde{B}_{A}(x), \tilde{B}_{A}(x \rightarrow y)\right\}=\left[\delta_{1}, \delta_{2}\right], \\
& J_{A}(y) \leq \max \left\{J_{A}(x), J_{A}(x \rightarrow y)\right\}=s,
\end{aligned}
$$

and so $M_{A}(y)=t, \tilde{B}_{A}(y)=\left[\delta_{1}, \delta_{2}\right]$ and $J_{A}(y)=s$. Hence, $y \in F$. Therefore, $F$ is a filter of $X$.

Proposition 3.25. Let $\mathcal{A}=\left(M_{A}, \tilde{B}_{A}, J_{A}\right)$ be an MBJ-neutrosophic filter of $X$. Then the sets $X_{M_{A}}=\left\{x \in X \mid M_{A}(x)=M_{A}(1)\right\}, X_{\tilde{B}_{A}}=\left\{x \in X \mid \tilde{B}_{A}(x)=\tilde{B}_{A}(1)\right\}, X_{J_{A}}=\left\{x \in X \mid J_{A}(x)=\right.$ $\left.J_{A}(1)\right\}$ are filters of $X$.

Proof. It is clear that $1 \in X_{M_{A}} \cap X_{\tilde{B}_{A}} \cap X_{J_{A}}$. Let $x, x \rightarrow y \in X_{M_{A}}$. Then $M_{A}(x \rightarrow y)=M_{A}(x)=$ $M_{A}(1)$. Since $\mathcal{A}$ is an MBJ-neutrosophic filter of $X$, we have

$$
M_{A}(1) \geq M_{A}(y) \geq \min \left\{M_{A}(x \rightarrow y), M_{A}(x)\right\}=\min \left\{M_{A}(1), M_{A}(1)\right\}=M_{A}(1) .
$$

Hence $M_{A}(y)=M_{A}(1)$ and so $y \in X_{M_{A}}$. Therefore $X_{M_{A}}$ is a filter of $X$. The proofs of other cases are similar.

Note. Since $1 \in X_{M_{A}} \cap X_{\tilde{B}_{A}} \cap X_{J_{A}}$, we get $X_{M_{A}} \cap X_{\tilde{B}_{A}} \cap X_{J_{A}} \neq \emptyset$ and by Proposition 3.25 . the sets $X_{M_{A}}, X_{\tilde{B}_{A}}$ and $X_{J_{A}}$ are filters of $X$, by using an MBJ-neutrosophic filter of $X$, for any $x, y \in X$, we can define a congruence relation on $X$ as follows:

$$
x \equiv_{\mathcal{A}} y \text { if and only if } x \rightarrow y, y \rightarrow x \in X_{M_{A}} \cap X_{\tilde{B}_{A}} \cap X_{J_{A}} .
$$

Corollary 3.26. Let $\frac{X}{\equiv_{\mathcal{A}}}=\left\{\frac{x}{\equiv_{\mathcal{A}}} \mid x \in X\right\}$. Define the operations $\simeq$ and $\sqcap$ on $\frac{X}{\equiv_{\mathcal{A}}}$ as follows:

$$
\frac{x}{\equiv_{\mathcal{A}}} \sqcap \frac{y}{\equiv_{\mathcal{A}}}=\frac{x \wedge y}{\equiv_{\mathcal{A}}} \text { and } \frac{x}{\equiv_{\mathcal{A}}} \simeq \frac{y}{\equiv_{\mathcal{A}}}=\frac{x \sim y}{\equiv_{\mathcal{A}}} .
$$

Hence $\left(\frac{X}{\equiv_{\mathcal{A}}}, \sqcap, \simeq, \frac{1}{\equiv_{\mathcal{A}}}\right)$ is an equality algebra.

\section{Conclusions}

In this paper, the notion of MBJ-neutrosophic sub-algebra and MBJ-neutrosophic filter on equality algebras are introduced and some equivalence definitions, properties and the relation between them are investigated. Also, by using the notion of MBJ-neutrosophic filter, a congruence relation is defined on equality algebra and we prove that the quotient structure that is made by this is an equality algebra.

In future work, we can study MBJ-neutrosophic notion on different kinds of filters such as (positive) implicative filters, fantastic filters, prime and maximal filters of equality algebras and investigate the quotient that is made by them. 


\section{References}

[1] M. Aaly Kologani, X.L. Xin, R.A. Borzooei, Y.B. Jun, Positive implicative equality algebras and equality algebras with some types, (submitted).

[2] K. Atanassov, Intutionistic fuzzy sets, Fuzzy Sets and Systems, 20(1) (1986), 87-96.

[3] R.A. Borzooei, M. Mohseni Takallo, F. Smarandache, Y.B. Jun, Positive implicative BMBJneutrosophic ideals in BCK-algebras, Neutrosophic Sets and Systems, 23 (2018), 126-141.

[4] R.A. Borzooei, M. Zarean, O. Zahiri, Involutive equality algebras, Soft Computing, 22 (2018), $7505-7517$.

[5] R.A. Borzooei, F. Zebardast, M. Aaly Kologani, Some types of filters in equality algebras, Categories and General Algebraic Structures with Applications, 7 (2017), 33-55.

[6] L.C. Ciungu, Internal states on equality algebras, Soft Computing, 19 (2015), 939-953.

[7] L. Henkin, Completeness in the theory of types, Journal of Symbolic Logic, 15 (1950), 81-91.

[8] S. Jenei, Equality algebras, Proceeding of CINTI 2010 conference-11th IEEE International Symposium on Computational Intelligence and Informatics, Budapest, Hungary, 2010.

[9] S. Jenei, Equality algebras, Studia Logica, 100 (2012), 1201-1209.

[10] S. Jenei, Kóródi, On the variety of equality algebras, Fuzzy Logic and Technology, (2011), $153-155$.

[11] Y.B. Jun, Neutrosophic subalgebras of several types in BCK/BCI-algebras, Annals of Fuzzy Mathematics and Informatics, 14(1) (2017), 75-86.

[12] Y.B. Jun, S.J. Kim, F. Smarandache, Interval neutrosophic sets with applications in $B C K / B C I$-algebra, Axioms, 7 (2018), 23.

[13] Y.B. Jun, F. Smarandache, H. Bordbar, Neutrosophic $\mathcal{N}$-structures applied to BCK/BCIalgebras, Information, 8 (2017), 128.

[14] Y.B. Jun, F. Smarandache, S.Z. Song, M. Khan, Neutrosophic positive implicative $\mathcal{N}$-ideals in BCK/BCI-algebras, Axioms, 7 (2018), 3.

[15] W.B.V. Kandasamy, F. Smarandache, Some neutrosophic algebraic structures and neutrosophic N-algebraic structures, Hexis, Phoenix, Arizona, 2006.

[16] W.B.V. Kandasamy, F. Smarandache, Neutrosophic rings, Hexis, Phoenix, Arizona, 2006.

[17] M. Khan, S. Anis, F. Smarandache, Y.B. Jun, Neutrosophic $\mathcal{N}$-structures and their applications in semigroups, Annals of Fuzzy Mathematics and Informatics, 14(6) (2017), 583-598.

[18] M. Mohseni Takallo, H. Bordbar, R.A. Borzooei, Y.B. Jun, BMBJ-neutrosophic ideals in BCK/BCI-algebras, Neutrosophic Sets and Systems, 27 (2019), 1-16.

[19] M. Mohseni Takallo, R.A. Borzooei, Y.B. Jun, MBJ-neutrosophic structures and its applications in BCK/BCI-algebras, Neutrosophic Sets and Systems, 23 (2018), 72-84.

[20] V. Novák, On fuzzy type theory, Fuzzy Sets and Systems, 149 (2005), 235-273. 
[21] V. Novák, B. De Baets, EQ-algebras, Fuzzy Sets and Systems, 160(20) (2009), 2956-2978.

[22] A.B. Saeid, Y.B. Jun, Neutrosophic subalgebras of BCK/BCI-algebras based on neutrosophic points, Annals of Fuzzy Mathematics and Informatics, 14(1) (2017), 87-97.

[23] F. Smarandache, Neutrosophy, neutrosophic probability, set, and logic, ProQuest Information and Learning, Ann Arbor, Michigan, USA, 105 p., 1998. http://fs.gallup.unm.edu/eBookneutrosophic s6.pdf (last edition online).

[24] F. Smarandache, A unifying field in logics. Neutrosophy: Neutrosophic probabilty, set and logic, Rehoboth: American Research Press, 1999.

[25] F. Smarandache, Neutrosophic set, a generalization of intuitionistic fuzzy sets, International Journal of Pure and Applied Mathematics, 24(5) (2005), 287-297.

[26] L.A. Zadeh, Fuzzy sets, Information and Control, 8(3) (1965), 338-353.

[27] F. Zebardast, R.A. Borzooei, M. Aaly Kologani, Results on equality algebras, Information Sciences, 381 (2017), 270-282. 